\title{
1 Separating overlapping bat calls with a bi-directional long short-term
}

\section{2 memory network}

\section{Using deep neural network to separate overlapping bat calls}

$4 \quad$ Kangkang Zhang ${ }^{1}$, Tong Liu ${ }^{1}$, Shengjing Song ${ }^{1}$, Xin Zhao ${ }^{1}$, Shijun Sun ${ }^{2}$, Walter

5 Metzner $^{3}$, Jiang Feng ${ }^{1,4^{*}}$, Ying Liu ${ }^{1 *}$

$6{ }^{1}$ Jilin Provincial Key Laboratory of Animal Resource Conservation and Utilization,

7 Northeast Normal University, Changchun, China. ${ }^{2}$ School of Environment, Northeast

8 Normal University, Changchun, China. ${ }^{3}$ Department of Integrative Biology and

9 Physiology, University of California, Los Angeles, California, USA. ${ }^{4}$ Collage of

10 Animal Science and Technology, Jilin Agricultural University, Changchun, China.

*Correspondence

E-mail: liuy252@nenu.edu.cn (YL)

14 E-mail: fengj@nenu.edu.cn (JF)

\section{Abstract}

Acquiring clear and usable audio recordings is critical for acoustic analysis of animal vocalizations. Bioacoustics studies commonly face the problem of overlapping signals, but the issue is often ignored, as there is currently no satisfactory solution. This study presents a bi-directional long short-term memory (BLSTM) network to

21 separate overlapping bat calls and reconstruct waveform audio sounds. The separation 
quality was evaluated using seven temporal-spectrum parameters. The applicability of this method for bat calls was assessed using six different species. In addition, clustering analysis was conducted with separated echolocation calls from each population. Results showed that all syllables in the overlapping calls were separated with high robustness across species. A comparison between the seven temporal-spectrum parameters showed no significant difference and negligible deviation between the extracted and original calls, indicating high separation quality. Clustering analysis of the separated echolocation calls also produced an accuracy of $93.8 \%$, suggesting the reconstructed waveform sounds could be reliably used. These results suggest the proposed technique is a convenient and automated approach for separating overlapping calls using a BLSTM network. This powerful deep neural network approach has the potential to solve complex problems in bioacoustics.

\section{Author summary}

In recent years, the development of recording techniques and devices in animal acoustic experiment and population monitoring has led to a sharp increase in the volume of sound data. However, the collected sound would be overlapped because of the existence of multiple individuals, which laid restrictions on taking full advantage of experiment data. Besides, more convenient and automatic methods are needed to cope with the large datasets in animal acoustics. The echolocation calls and communication calls of bats are variable and often overlapped with each other both in the recordings from field and laboratory, which provides an excellent template for research on animal sound separation. Here, we firstly solved the problem of 
44 overlapping calls in bats successfully based on deep neural network. We built a

45 network to separate the overlapping calls of six bat species. All the syllables in

46 overlapping calls were separated and we found no significant difference between the

47 separated syllables with non-overlapping syllables. We also demonstrated an instance

48 of applying our method on species classification. Our study provides a useful and

49 efficient model for sound data processing in acoustic research and the proposed

50 method has the potential to be generalized to other animal species.

\section{$51 \quad$ Introduction}

52 The structural identification of vocal units is essential in animal acoustic studies for

53 sound feature analysis, sound emitter recognition, and species identification and

54 monitoring. However, wild animal monitoring, both in the field and in the laboratory,

55 often involves problems caused by the overlapping of different vocal units in time and

56 frequency space, which prevents the components from being suitable for parameter

57 analysis. As a result, the separation of overlapping sounds is an important task in

58 bioacoustic signal processing. However, existing analysis software often struggles to

59 process overlapping calls and previous research on the acoustic identification of

60 animals primarily focuses on extracting target signals from background noise for

61 species classification or population monitoring [1-4]. The process of separating

62 overlapping calls from mixed sounds has received little attention to date and

63 researchers conventionally abandon sounds that overlap in both time and frequency,

64 requiring an extension of the experimental period to obtain sufficient non-overlapping

65 recordings $[5,6]$. As such, an effective method for successfully and automatically 
66

67

68

69

70

71

72

73

74

75

76

77

78

79

80

81

82

83

84

85

86

87

separating overlapping calls would be of significant interest and benefit to animal researchers.

Previous studies using deep neural networks have produced promising results for automated sound recognition in complex acoustic environments for animal species recognition and classification [6-8]. However, in this study, we consider the more difficult task of separating different types of syllables from overlapping calls and reconstructing sound waves from these separated signals. Existing techniques used for animal sound separation often require prohibitive quantities of labelled data. For example, multiple-instance machine learning (MIML) algorithms were proposed for use in sound feature extraction and species identification in birds [1]. However, this technique requires a cropped mask of a signal segment (without overlap) in order to extract each syllable.

Deep learning networks have been applied to bioacoustic studies but have primarily been used for classification. For instance, convolutional bidirectional recurrent neural networks (CBRNNs) have been used to identify the presence of bird calls in audio samples [4]. Acoustic features were learned by the network (a classifier) and the presence or absence of a bird call was output as an indicator. Convolutional neural networks (CNNs) have been used to predict the presence of a search-phase bat echolocation call in spectrograms. This binary classification problem was used to detect the presence of bats [2]. To our knowledge, the use of deep learning techniques to separate animal calls that overlap in both time and frequency space has yet to be reported. 
$88 \quad$ Multiple studies have been conducted using deep learning-based supervised speech

89 separation with humans. Early systems included shallow models that performed a

90 linear transformation of given mixture features during the prediction time interval.

91 This has included Gaussian mixture models [9], support vector machines [10], and

92 non-negative matrix factorization [11]. However, in real-world scenarios, the mapping

93 relationship between mixture signals and sources is typically a nonlinear

94 transformation. Nonlinear models, such as deep neural networks (DNNs), are

95 therefore highly applicable because of their ability to identify nonlinear structures in

96 audio signals [12-14]. Additionally, recurrent neural networks (RNNs) that exhibit the

97 temporal behavior of a time sequence can be trained to predict time-frequency masks

98 for target signals and separate sources from a mixed waveform [15]. Specifically, long

99 short-term memory (LSTM) networks, a variation of RNN models that exhibit strong

100 learning capabilities and simple construction, have been widely used for word and

101 continuous speech recognition [16-18]. By concatenating two separate LSTM

102 networks, bidirectional LSTMs (BLSTMs) can predict each element of a sequence

103 based on past and future context and can naturally account for the temporal dynamics

104 of speech. These models are typically faster and more accurate than standard RNNs in

105 frame-by-frame phoneme classification [19]. In addition, the BLSTM network can

106 compensate for exploding and vanishing gradient issues that can occur during the

107 training of standard RNN models [20]. At present, BLSTMs have achieved

108 state-of-the-art performance for speech recognition $[14,21]$, natural language

109 processing [22, 23], and speaker-independent speech separation [24]. As such, a 
110

BLSTM model was selected in this study for overlapping bat call separation.

Echolocating bats have two vocal repertoires, stereotypical echolocation calls for orientation and a variety of communication calls for social activities [25-27]. Recordings from both field and laboratory studies indicate that utterances from individual bats often overlap in both time and frequency, which provides an excellent template for research on overlapping sound separation in animals. The primary objective of this study is to develop a technique for separating two target signals (echolocation and socialization calls) from mixtures of acoustic sounds. Although deep leaning has been employed in the acoustic classification of multiple species, including nonhuman primates [28], birds [4], whales [5], and bats [2, 3], the goal of the present study is distinct from these previous cases in which deep neural networks were primarily used as classifiers.

Both overlapping and non-overlapping calls (of both echolocation and communication types) were recorded from each of the collected bat species studied in our previous work. We developed a BLSTM network and used the recorded non-overlapping calls to train the model. Recorded overlapping calls were input to the trained model and separated. Independent sound files were then reconstructed for each separated signal. The correctness of these separated signals was measured by comparing the temporal-spectrum parameters between separated calls and the initially recorded (non-overlapping) calls from each species. Finally, clustering analysis was conducted to classify the bats using separated echolocation calls, which provided a practical application of the proposed technique. 
132

134

136

137

138

\section{Results}

The proposed algorithm performed well and achieved high accuracy in separating overlapping calls for each of the six species. The BLSTM model was iteratively trained until the training and validation losses reached a minimum. Loss is a summation of errors made with each sample in the training or validation sets and measures how well the model adapts during optimization. Training loss for this model decreased significantly in the first epoch. The validation loss function tended toward an asymptotic value, indicating the training algorithm had converged (S2 Fig). The BLSTM model converged slightly faster when training with CF bat samples (as opposed to FM samples).

All echolocation and communication calls in the overlapping signals were correctly extracted during the separation procedure, regardless of their pulse duration or energy characteristics (see Table 1 and Fig 1). In addition, low-intensity FM components in echolocation pulses were successfully extracted from three CF bat species (Figs 1d, 1e, and 1f).

Table 1. Separation results.

\begin{tabular}{|c|c|c|c|c|c|}
\hline Species & Call type & $\begin{array}{c}\text { Number of } \\
\text { syllable types }\end{array}$ & $\begin{array}{l}\text { Number of } \\
\text { syllables in } \\
\text { overlapping } \\
\text { calls }\end{array}$ & $\begin{array}{c}\text { Number of } \\
\text { overlapping } \\
\text { syllables }\end{array}$ & $\begin{array}{c}\text { Number of } \\
\text { separated } \\
\text { syllables }\end{array}$ \\
\hline \multirow{2}{*}{$\begin{array}{c}\text { Rhinolophus } \\
\text { ferrumequinum }\end{array}$} & Echolocation & 1 & 14 & 14 & 14 \\
\hline & Communication & 4 & 8 & 8 & 8 \\
\hline \multirow{2}{*}{$\begin{array}{c}\text { Vespertilio } \\
\text { sinensis }\end{array}$} & Echolocation & 1 & 21 & 13 & 13 \\
\hline & Communication & 4 & 8 & 8 & 8 \\
\hline \multirow{2}{*}{$\begin{array}{c}\text { Hipposideros } \\
\text { armiger }\end{array}$} & Echolocation & 1 & 28 & 19 & 19 \\
\hline & Communication & 6 & 13 & 13 & 13 \\
\hline Myotis & Echolocation & 1 & 54 & 36 & 36 \\
\hline
\end{tabular}




\begin{tabular}{|c|c|c|c|c|c|}
\hline macrodactylus & Communication & 6 & 15 & 15 & 15 \\
\hline \multirow{2}{*}{$\begin{array}{c}\text { Rhinolophus } \\
\text { pusillus }\end{array}$} & Echolocation & 1 & 42 & 30 & 30 \\
\cline { 2 - 6 } & Communication & 6 & 10 & 10 & 10 \\
\hline \multirow{2}{*}{ Ia io } & Echolocation & 1 & 26 & 16 & 16 \\
\cline { 2 - 6 } & Communication & 4 & 11 & 11 & 11 \\
\hline
\end{tabular}

Fig 1. Spectrograms from original recordings of overlapping calls and calls separated by

the BLSTM network. The first graph represents each line of the original overlapping calls and the second and third graphs show the separated echolocation and communication calls, respectively.

A comparison of seven temporal-spectrum parameters from the separated calls and the original recorded non-overlapping calls showed no significant differences (Fig 2 and S3 Table). In addition, parameter deviations in separated calls and original non-overlapping calls showed minimal RMSE values for both echolocation and communication signals (Fig 3 and Fig 4). Clustering analysis performed with separated echolocation calls produced an accuracy of 93.8\% across species (Fig 5).

Fig 2. Comparisons between the separated and original calls. Two principle respectively.

Fig 3. A comparison of deviations for separated and original echolocation calls. 
167 each parameter and the horizontal axis represents the number of syllables measured.

168 The red triangles represent separated calls and the blue dots represent original calls.

169 Abbreviations include duration (duration), Fstart (starting frequency), Fend (ending

170 frequency), Fpeak (peak frequency), Fmin (minimum frequency), Fmax (maximum

171 frequency), and bandw (bandwidth).

172 Fig 4. A comparison of deviations in separated and original communication calls.

173 The RMSE value is shown under each plot. The vertical axes and abbreviations are

174 the same in Fig 3.

175 Fig 5. Clustering analysis for six bat species based on their separated

176 echolocation calls. Overlapping echolocation signals cannot be used for species

177 identification until after separation.

178

179

180

181

182

183

184

185

186

187

188

\section{Discussion}

The BLSTM network used in the present study achieved high accuracy in separating overlapping echolocation and communication calls from bats. The training and validation loss for the model also exhibited fast convergence and high robustness for bat vocalizations. In particular, the separated calls extracted by the proposed algorithm were reconstructed as waveform files with nearly the same quality as the non-overlapping calls, suggesting BLSTM networks to be useful tools for separating signals in future bioacoustic research, such as sound analysis, acoustic identification, species classification, and wild animal monitoring.

It was difficult to compare the performance of this algorithm with that of previous 
189

190

studies, primarily because of differences in the experimental procedure. However, a comparison of temporal-spectrum parameters between separated calls and non-overlapping calls was included as an evaluation metric. The seven parameters used in this study are commonly used in bat studies to describe the temporal-spectral features of syllables [26, 29]. Statistical results for this comparison showed no significant differences and small deviations in parameters between separated calls and original recordings, indicating the system was able to separate calls without affecting syllable quality. In addition, clustering analysis conducted with reconstructed echolocation calls was highly accurate $(93.8 \%)$ for species classification, indicating that calls separated from overlapping signals could be used to synthesize initial data. The BLSTM network exhibited good performance across all six bat species using both narrow and broad time-frequency calls. It also successfully separated different syllable types from both overlapping echolocation and communication calls (Table 1, Fig 1). No species-specific a priori knowledge or particular acoustic sensor was directly encoded into the system, making it generalizable to other animal populations with additional training data. Although the dichotomy between communication and echolocation calls is relatively drastic, the proposed separation system has potential applications for other species, as such mixtures are very common in bats. In the future, more complex emitter-independent separation could be conducted using the proposed system, such as combinations of echolocation or social calls from other animals.

While deep learning models generally perform better when provided with more data, training with bat calls requires fewer samples than human speech separation, in which 
211 available training sets can exceed hundreds of hours [13]. One possible reason for this

212 may be the high signal-to-noise ratio (SNR) of bat sounds recorded with high-quality

213 ultrasound devices. Previous studies have indicated that a high SNR can improve

214 separation accuracy [30] and our results suggest this model was suitable for use with

215 small, high-quality datasets. Although the sound data in this study were sampled in

216 controlled lab conditions, producing recordings that were essentially free of

217 background noise, acoustic analysis software could potentially optimize the separation

218 further by excluding any background noise that was present in the signal.

219 Future studies will also assess the performance of this network for other animal

220 species. Stereotypical patterns and clearly classifiable syllables have been observed in

221 the vocalizations of birds, non-human primates, whales, dolphins, and several other

222 species [31-33]. Features used in the proposed BLSTM were log spectral magnitudes,

223 which can be acquired from any vocal sound. This could potentially lead to robust

224 software that is not specific to a certain species or task. The model could also be

225 generalized to other animals, though limitations may exist. In addition to the quality

226 and quantity of training samples, hyper-parameters must be tuned in accordance with

227 the data $[34,35]$.

228 Conclusion

229 A sound separation model was proposed for extracting bat calls, achieving

230 excellent results. This is the first experimental evidence that the BLSTM model is

231 suitable for separating overlapping bioacoustic signals. These results provided a new

232 source for sound data analysis in animal acoustics research, which may contribute to 
233

234

235

sample sizes and improve efficiency. This study also demonstrates the potential of deep neural networks for applications to animal vocalization research, including species classification and speech separation.

\section{Materials and Methods}

\section{Sound recording and data preparation}

Species selection and sound sources. Echolocation calls from bats are primarily composed of constant frequency $(\mathrm{CF})$ components and frequency modulated (FM) components. Social calls are composed of CF, FM, and noise-burst (NB) components. FM calls have short pulse durations and wide bandwidths. As such, they overlap with social calls less in time but more in frequency. In contrast, CF calls have long pulse durations and narrow bandwidths. They overlap with social calls more in time but less in frequency. In consideration of the varied overlapping patterns found in bat calls, we selected both CF bats (Rhinolophus ferrumequinum, Hipposideros armiger, and Rhinolophus pusillus) and FM bats (Vespertilio sinensis, Myotis macrodactylus, and Ia io) to test the separation capabilities of the proposed network, including six different species to test method generalizability.

Source sound files from $V$. sinensis, M. macrodactyllus, $R$. ferrumequinum, $R$. pusillus, and $H$. armiger were collected from previous studies in our lab (S1 Table). Sound files for Ia io were selected from unpublished data as follows. Bats captured from the field were housed in a husbandry room with abundant food and fresh water.

During each sound recording experiment, 4-5 bats were transferred to a temporary cage. Sound recordings were collected using the Avisoft UltraSoundGate 116H 
255 (Avisoft Bioacoustics, Berlin, Germany) and a condenser ultrasound microphone

256 (CM16/CMPA, Avisoft Bioacoustics). The sampling frequency was set to $375 \mathrm{kHz}$ at

25716 bits. The recording experiment lasted five days in order to acquire a sufficient

258 number of recordings, beginning at 18:00 and finishing at 6:00 the following morning.

259 S1 Table shows sample numbers and locations for the bats, as well as the total

260 duration of sound files selected for the study. All experimental procedures complied

261 with the ABS/ASAB guidelines for the Use of Animals in Research and were

262 approved by the Committee on the Use and Care of Animals at the Northeast Normal

263 University (approval number: NENU-W-2010-101).

264 Sound analysis. The total duration of recorded sound files (i.e., original recording

265 files) used for each bat species is shown in S1 Table. We employed Avisoft-SASLab

266 Pro (Version 5.2.12, Avisoft Bioacoustics, Berlin, Germany) to identify

267 non-overlapping and overlapping syllables in echolocation and communication calls.

268 These syllables and calls were described and classified following the nomenclature

269 developed by Kanwal, Matsumura (36) and Ma, Kobayasi (37). The recorded

270 non-overlapping calls were used for preparing training files of each call type and the

271 recorded overlapping calls were used for separation.

272 Data preparation. Supervised machine learning algorithms use training samples to

273 "learn" the steps required for completing a task. The training phase in this study

274 involved preparing clear and non-overlapping echolocation and communication calls,

275 selected from original recording sounds. In this process, the BLSTM network learned

276 features found in both call types. 
277 Training samples consisted of randomly selected non-overlapping syllables in

278 echolocation and communication calls from each bat species (in the original

279 recordings), with signal-to-noise ratios (SNRs) above $-20 \mathrm{~dB}$. The echolocation

280 training files contained 1,300-6,240 pulses and the communication training files

281 contained 780-1,800 syllables (S1 Table). Although the quantity of selected syllables

282 varied between studies, the data was sufficient for model training. Efforts were made

283 to include roughly equivalent quantities of each syllable type. Time intervals between

284 syllables in the training files were consistent with those of the original recordings. The

285 lengths of training files for echolocation and communication calls were the same for

286 each bat species (S1 Table).

287 Model training and call separation

288 Model structure and training stage. We developed a network with four BLSTM

289 layers, followed by one feedforward layer (Fig 6). Each BLSTM layer included one

290 forward and one backward basic LSTM layer, both of which were added with dropout

291 functions (tensorflow.nn.rnn_cell.DropoutWrapper). Each BLSTM layer contained

292300 hidden cells and the feedforward layer corresponded to the embedding dimension

293 (i.e., a 3D matrix with depth $\mathrm{N}=40$ in this experiment). Stochastic gradient descent

294 with a momentum of 0.9 and a fixed learning rate of $10^{-3}$ was used for training. The

295 tanh activation function and the Adam optimizer were adopted to support adaptive

296 learning rates and faster convergence. The structure and hyper-parameters for the

297 model were designed based on the work of Hershey, Chen (21).

298 Fig 6. The BLSTM model architecture and workflow graph. 
The model was trained using the files for one bat species in each trial.

300 Echolocation and communication call training files were loaded using the librosa

301 (version 0.6.2) Python package. Frames from the two sound files were read and added

302 together to create sound mixtures. Sound features used for training (log spectral

303 magnitudes) were extracted from this mixture. The extraction process was completed

304 using a short-time Fourier transform (STFT) with a Hamming window (length of 512

305 and shift of 256).

306 The mixture from each bat species was then segmented into 100-frame samples,

307 all of which were divided into a training set and a validation set using a ratio of 2:1

308 (see S1 Table for detailed sample quantities). The training set, validation set, and

309 indicator labels were combined and input to the model. The validation set was used to

310 optimize tuning parameters and evaluate call separation performance. Indicator labels

311 were set to 0 or 1 , representing the two types of calls in the mixture. Ideal binary

312 masks were used to train the network and gradients were calculated using shuffled

313 mini-batches (batch size of 128) from larger segments.

314 The output of this model was a set of embeddings that included learned features

315 for both echolocation and communication calls. In this framework, the deep network

316 assigned embedding vectors to each time-frequency bin in the spectrogram. The

317 network then minimized the distance between embeddings dominated by the same

318 call type in each bin while maximizing the distance between embeddings dominated

319 by different call types. The output was then compared with the validation set and 320 indicator labels to calculate loss, which was back propagated from the output to the 
321 input through each layer. Model weights and parameters were then updated based on

322 the calculated loss and training was completed after sufficient iteration epochs.

323 Separation stage. In this stage, overlapping echolocation and communication calls

324 were randomly selected from the original recordings to create a sound file of test sets,

325 used for separation. The log spectral magnitudes of the overlapping calls were then

326 extracted, combined into samples, and input to the trained model. The phases of calls

327 extracted from the sound files were also saved for use in sound reconstruction. The

328 trained model then output embeddings for each segment (100 frames) in a process

329 similar to the training stage. Embeddings were clustered using the k-means method

330 from Scikit-learn (Version 0.20.0) to produce time-frequency masks. The number of

331 clusters corresponded to the number of call types in the mixture (2 - echolocation and

332 communication). These masks and the clustering method were then used to determine

333 which parts of each segment in the overlapped calls would be preserved or neglected

334 based on their correspondence to each call type. For example, if the maximum

335 magnitudes were more likely to belong to echolocation calls, the related mask values

336 were set to 1 and the others were set to 0 , allowing the echolocation calls to be separated

337 correctly. Finally, output calls were reconstructed using the inverse fast Fourier

338 transform (IFFT) function numpy.fft.ifft in NumPy (Version 1.15.1). The IFFT

339 transformed the magnitude into a wave using phase information saved at the beginning

340 of the separation stage. The model produced two waveform files, each containing one

341 call type. Additional detail concerning the sound separation algorithms can be found in

342 the work of Hershey (2016). 
343

344

345

346

347

348

349

350

351

352

353

354

355

356

357

358

\section{9}

360

361

362

363

364

\section{Model evaluation}

The quality of reconstructed echolocation and communication calls was assessed by comparing their temporal-spectrum parameters to the non-overlapping calls selected from the original recording files (excluding training data). Avisoft-SASLab Pro was used for automatic parameter measurements of duration, bandwidth, peak frequency, minimum frequency, maximum frequency, starting frequency, and ending frequency. A t-SNE (t-distributed stochastic neighbor embedding - R3.6.1 package) analysis was adopted for dimensionality reduction. Two dimensions were extracted from these seven parameters for original and separated syllables and compared with one-way ANOVA (aov in R3.6.1) or two-sided Wilcoxon signed-rank tests (wilcox.test in R3.6.1), depending on their fit to a normal Gaussian distribution. The significance level was set to 0.05 for all tests. We adopted the root mean square error (RMSE) to measure and avoid obscuring individual variations between reconstructed and original calls. Clustering analysis was conducted using the reconstructed echolocation calls from the six bat species, to assess whether the separated calls could be further used in species classification.

\section{Acknowledgements}

We are grateful to Dr. Yanhong Xiao of the Experimental Center of the School of Environment at Northeast Normal University, for her assistance in acquiring the experimental materials. We thank LetPub (www.letpub.com) for its linguistic assistance during the preparation of this manuscript.

\section{Author contributions}


365 Conceptualization: Kangkang Zhang, Walter Metzner.

366 Data curation: Tong Liu, Shengjing Song, Xin Zhao.

367 Formal Analysis: Kangkang Zhang, Tong Liu.

368 Methodology: Kangkang Zhang, Ying Liu, Jiang Feng.

369 Software: Kangkang Zhang, Shijun Sun.

370 Funding acquisition: Ying Liu, Jiang Feng, Walter Metzner.

371 Supervision: Jiang Feng.

372 Visualization: Kangkang Zhang, Tong Liu.

373 Writing - original draft: Kangkang Zhang, Ying Liu.

374 Writing - review \& editing: Ying Liu, Walter Metzner.

375 Competing interests

376 The authors have declared that no competing interests exist. 
377

378

379

380

381

382

383

384

385

386

387

388

389

390

391

392

393

394

395

396

397

398

399

400

401

402

403

404

405

406

407

408

409

410

411

412

413

414

415

416

417

418

419

\section{References}

1. Briggs F, Lakshminarayanan B, Neal L, Fern XZ, Raich R, Hadley SJK, et al. Acoustic classification of multiple simultaneous bird species: A multi-instance multi-label approach. 2012;131(6):4640-50. doi: 10.1121/1.4707424.

2. Aodha OM, Gibb R, Barlow KE, Browning E, Firman M, Freeman R, et al. Bat detective-Deep learning tools for bat acoustic signal detection. PLOS Computational Biology. 2018;14(3):156869.

3. Walters CL, Freeman R, Collen A, Dietz C, Brock Fenton M, Jones G, et al. A continental-scale tool for acoustic identification of European bats. Journal of Applied Ecology. 2012;49(5):1064-74. doi: https://doi.org/10.1111/j.1365-2664.2012.02182.x.

4. Adavanne S, Drossos K, Cakir E, Virtanen T. Stacked convolutional and recurrent neural networks for bird audio detection. european signal processing conference. 2017:1729-33. doi: 10.23919/EUSIPCO.2017.8081505.

5. Shamir L, Yerby C, Simpson R, Benda-Beckmann AMv, Tyack P, Samarra F, et al. Classification of large acoustic datasets using machine learning and crowdsourcing: Application to whale calls. The Journal of the Acoustical Society of America. 2014;135(2):953-62. doi: https://doi.org/10.1121/1.4861348.

6. Priyadarshani N, Marsland S, Castro I. Automated birdsong recognition in complex acoustic environments: a review. Journal of Avian Biology. 2018;49(5):jav-01447. doi: https://doi.org/10.1111/jav.01447.

7. Redgwell RD, Szewczak JM, Jones G, Parsons S. Classification of echolocation calls from 14 species of bat by support vector machines and ensembles of neural networks. Algorithms. 2009;2(3):907-24.

8. Sprengel E, Jaggi M, Kilcher Y, Hofmann T, editors. Audio based bird species identification using deep learning techniques. LifeCLEF 2016; 2016.

9. Kim G, Lu Y, Hu Y, Loizou PC. An algorithm that improves speech intelligibility in noise for normal-hearing listeners. The Journal of the Acoustical Society of America. 2009;126(3):1486-94. doi: 10.1121/1.3184603.

10. Wang Y, Wang D. Towards Scaling Up Classification-Based Speech Separation. IEEE Transactions on Audio, Speech, and Language Processing. 2013;21(7):1381-90. doi: 10.1109/TASL.2013.2250961.

11. Grais EM, Erdogan H, editors. Spectro-temporal post-smoothing in NMF based single-channel source separation. Signal Processing Conference (EUSIPCO), 2012 Proceedings of the 20th European; 2012: IEEE.

12. Nugraha AA, Liutkus A, Vincent E. Deep Neural Network Based Multichannel Audio Source Separation. Signals Commun Techn. 2018:157-85. doi: 10.1007/978-3-319-73031-8_7. PubMed PMID: WOS:000441018800008.

13. Wang D, Chen J. Supervised Speech Separation Based on Deep Learning: An Overview. IEEE Transactions on Audio, Speech, and Language Processing. 2018;26(10):1702-26. doi: 10.1109/Taslp.2018.2842159. PubMed PMID: WOS:000438718300001.

14. Marchi E, Ferroni G, Eyben F, Gabrielli L, Squartini S, Schuller B, editors. Multi-resolution linear prediction based features for audio onset detection with bidirectional LSTM neural networks. 2014 IEEE international conference on acoustics, speech and signal processing (ICASSP); 2014: IEEE.

15. Weninger F, Hershey JR, Le Roux J, Schuller B, editors. Discriminatively trained recurrent neural 
420

421

422

423

424

425

426

427

428

429

430

431

432

433

434

435

436

437

438

439

440

441

442

443

444

445

446

447

448

449

450

451

452

453

454

455

456

457

458

459

460

461

462

463

networks for single-channel speech separation. Proceedings 2nd IEEE Global Conference on Signal and Information Processing, GlobalSIP, Machine Learning Applications in Speech Processing Symposium, Atlanta, GA, USA; 2014.

16. Eck D, Graves A, Schmidhuber J. A new approach to continuous speech recognition using LSTM recurrent neural networks. Technical Report. 2003.

17. Beringer $\mathrm{N}$, editor Human language acquisition methods in a machine learning task. Eighth International Conference on Spoken Language Processing; 2004.

18. Graves A, Beringer N, Schmidhuber J, editors. A Comparison Between Spiking and Differentiable Recurrent Neural Networks on Spoken Digit Recognition. international conference on modelling identification and control; 2004.

19. Graves A, Schmidhuber J. Framewise phoneme classification with bidirectional LSTM and other neural network architectures. Neural Networks. 2005;18(5):602-10. doi: https://doi.org/10.1016/j.neunet.2005.06.042.

20. Makino S. Audio Source Separation: Springer; 2018.

21. Hershey JR, Chen Z, Roux JL, Watanabe S, editors. Deep clustering: Discriminative embeddings for segmentation and separation. 2016 IEEE International Conference on Acoustics, Speech and Signal Processing (ICASSP); 2016 20-25 March 2016.

22. Wöllmer M, Eyben F, Graves A, Schuller B, Rigoll GJCC. Bidirectional LSTM networks for context-sensitive keyword detection in a cognitive virtual agent framework. 2010;2(3):180-90.

23. Huang Z, Xu W, Yu K. Bidirectional LSTM-CRF models for sequence tagging. arXiv preprint arXiv:150801991. 2015.

24. Li C, Zhu L, Xu S, Gao P, Xu B, editors. CBLDNN-Based Speaker-Independent Speech Separation Via Generative Adversarial Training. international conference on acoustics, speech, and signal processing; 2018.

25. Kunz TH, Fenton MB. Bat ecology: University of Chicago Press; 2005.

26. Gillam E, Fenton MB. Roles of acoustic social communication in the lives of bats. Bat Bioacoustics: Springer; 2016. p. 117-39.

27. Luo B, Huang X, Li Y, Lu G, Zhao J, Zhang K, et al. Social call divergence in bats: a comparative analysis. Behavioral Ecology. 2017;28(2):533-40. doi: 10.1093/beheco/arw184.

28. Pozzi L, Gamba M, Giacoma C. The Use of Artificial Neural Networks to Classify Primate Vocalizations: A Pilot Study on Black Lemurs. Am J Primatol. 2010;72(4):337-48. doi: 10.1002/ajp.20786. PubMed PMID: WOS:000276124300007.

29. Jin L, Wang J, Zhang Z, Sun K, Kanwal JS, Feng J. Postnatal development of morphological and vocal features in Asian particolored bat, Vespertilio sinensis. Mammalian Biology. 2012;77(5):339-44. doi: 10.1016/j.mambio.2012.05.001. PubMed PMID: WOS:000309023900005.

30. Weng C, Yu D, Seltzer ML, Droppo J. Deep neural networks for single-channel multi-talker speech recognition. IEEE Transactions on Audio, Speech, and Language Processing. 2015;23(10):1670-9. doi: 10.1109/Taslp.2015.2444659. PubMed PMID: WOS:000356518100012.

31. Naguib M, Riebel K. Bird song: a key model in animal communication. Encyclopedia for language and linguistics. 2006;2:40-53.

32. Filatova OA, Deecke VB, Ford JKB, Matkin CO, Barrett-Lennard LG, Guzeev MA, et al. Call diversity in the North Pacific killer whale populations: implications for dialect evolution and population history. Animal behaviour. 2012;83(3):595-603. doi: https://doi.org/10.1016/j.anbehav.2011.12.013. 
464

465

466

467

468

469

470

471

472

473

474

475

476

\section{Supporting information}

478

479

480

481

482

483

484 PubMed PMID: 16418857.

33. Herzing DL. Clicks, whistles and pulses: Passive and active signal use in dolphin communication. Acta Astronautica. 2014;105(2):534-7. doi: 10.1016/j.actaastro.2014.07.003.

34. Lecun Y, Bengio Y, Hinton G. Deep learning. Nature. 2015;521(7553):436. doi: 10.1038/nature14539. PubMed PMID: 26017442.

35. Goodfellow I, Bengio Y, Courville A. Deep Learning: The MIT Press; 2016.

36. Kanwal JS, Matsumura S, Ohlemiller K, Suga N. Analysis of acoustic elements and syntax in communication sounds emitted by mustached bats. The Journal of the Acoustical Society of America. 1994;96(3):1229-54. doi: https://doi.org/10.1121/1.410273.

37. Ma J, Kobayasi K, Zhang S, Metzner W. Vocal communication in adult greater horseshoe bats, Rhinolophus ferrumequinum. Journal of comparative physiology A, Neuroethology, sensory, neural, and behavioral physiology. 2006;192(5):535-50. doi: https://doi.org/10.1007/s00359-006-0094-9.

\section{S1 Table. A summary of calls used for model training.}

\section{S2 Fig. Training loss and validation loss during model training.}

\section{S3 Table. Statistical comparisons of principle components extracted from seven}

parameters. No significant differences were observed between parameters for separated and original syllables. A one-way ANOVA was used to test the normal distributed data and a two-sided Wilcoxon signed-rank test was used to assess the data that did not conform well to a normal distribution. 


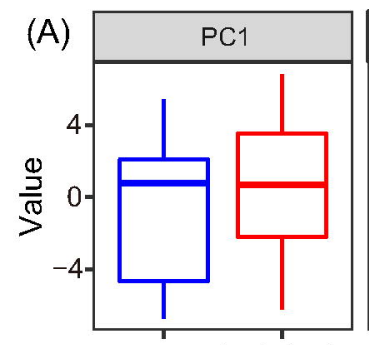

extracted original extracted original ANOVA: $d f=34 \quad$ ANOVA: $d f=34$ $\mathrm{F}=1.49 p=0.23 \quad \mathrm{~F}=0.99 p=0.33$

Vespertilio sinensis

(D)
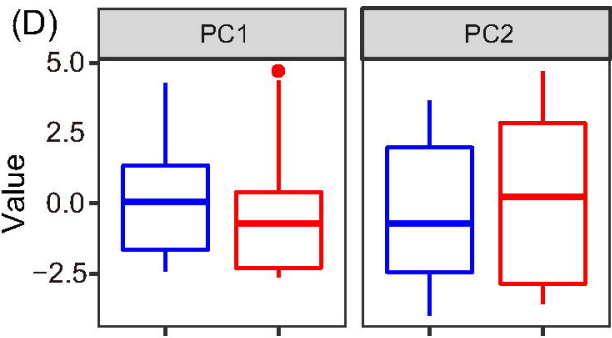

extracted original extracted original

Wilcoxon: $p=0.38$ Wilcoxon: $p=0.87$

Rhinolophus ferrumequinum

(G)

$\frac{0}{\frac{N}{N}}$

$\frac{\frac{1}{2}}{\frac{\pi}{7}}$

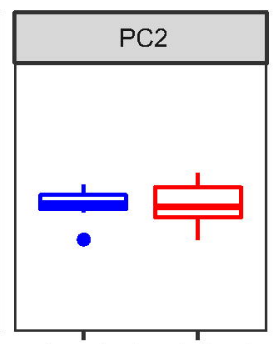

extracted original extracted original

ANOVA: $d f=14 \quad$ ANOVA: $d f=14$

$\mathrm{F}=0.43 p=0.52 \quad \mathrm{~F}=0.002 p=0.97$

Vespertilio sinensis

(J)

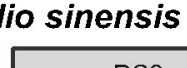

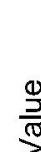

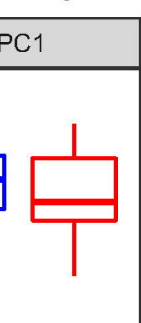

$-600$

extracted original

ANOVA: $d f=14$

$\mathrm{F}=0.05 p=0.82$

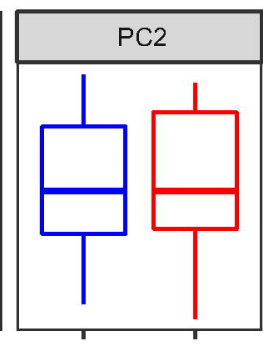

extracted original

ANOVA: $d f=14$

$\mathrm{F}=0 \quad p=0.99$
Rhinolophus ferrumequinum

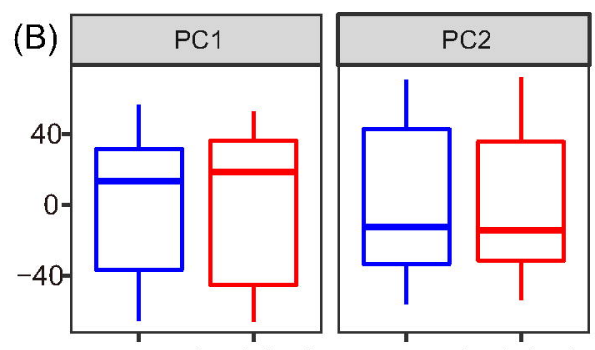

extracted original extracted original

Wilcoxon: $p=0.82$ Wilcoxon: $p=0.78$

la io
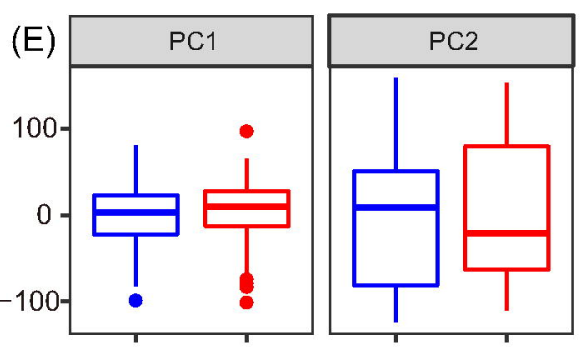

extracted original extracted original

Wilcoxon: $p=0.44$ Wilcoxon: $p=0.67$

Hipposideros armiger
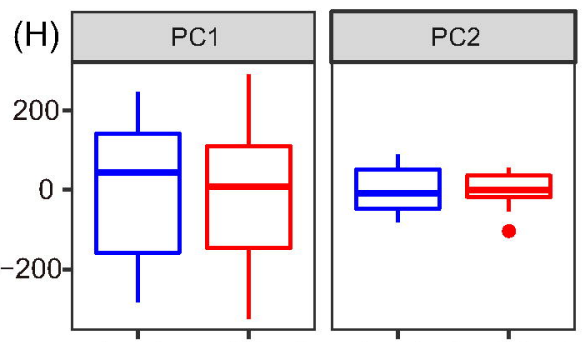

extracted original extracted original

ANOVA: $d f=20 \quad$ ANOVA: $d f=20$

$\mathrm{F}=0.002 p=0.97 \quad \mathrm{~F}=0.002 p=0.97$

la io

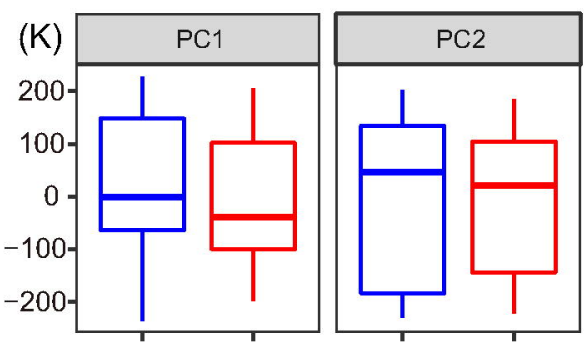

extracted original extracted original ANOVA: $d f=24$

$\mathrm{F}=0.17 p=0.69$

Wilcoxon: $p=0.98$

Hipposideros armiger
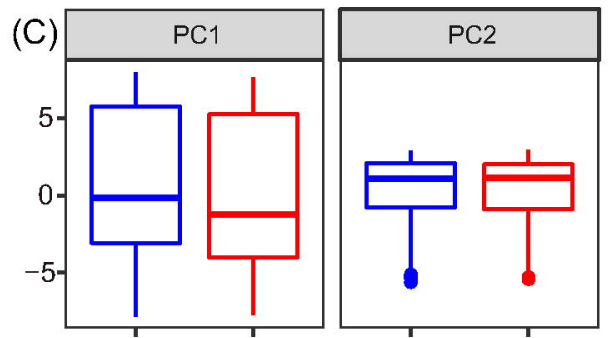

extracted original

extracted original

Wilcoxon: $p=0.20$ Wilcoxon: $p=0.94$

\section{Mvotis macrodactylus}

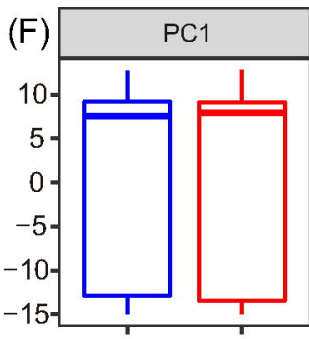

extracted original

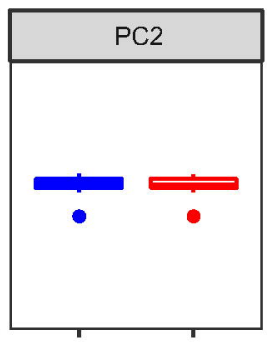

extracted original

Wilcoxon: $p=0.81$ Wilcoxon: $p=0.31$

Rhinolophus pusillus
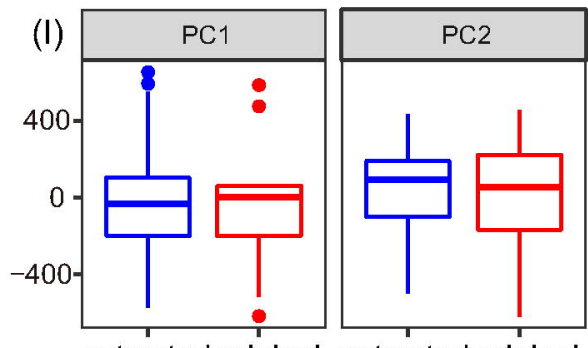

extracted original extracted original

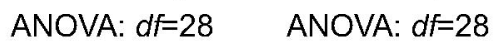

$\mathrm{F}=0.05 p=0.82 \quad \mathrm{~F}=0.02 p=0.89$

Mvotis macrodactylus

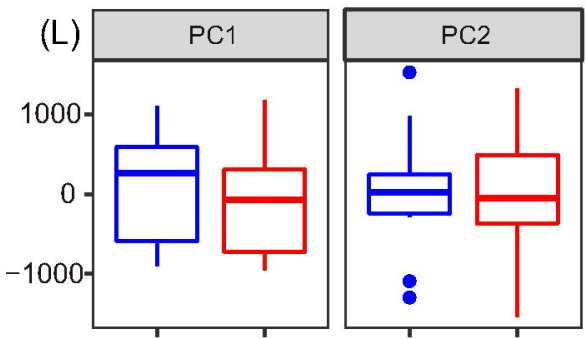

extracted original extracted original

ANOVA: $d f=18 \quad$ ANOVA: $d f=18$

$\mathrm{F}=0.33 p=0.58 \quad \mathrm{~F}=0.01 p=0.91$

Rhinolophus pusillus 
bioRxiv preprint doi: https://doi.org/10.1101/2019.12.15.876656; this version posted December 15, 2019. The copyright holder for this preprint

(which was not certified by peer review) is the author/funder, who has granted bioRxiv a license to display the preprint in perpetuity. It is made available under aCC-BY 4.0 International license.

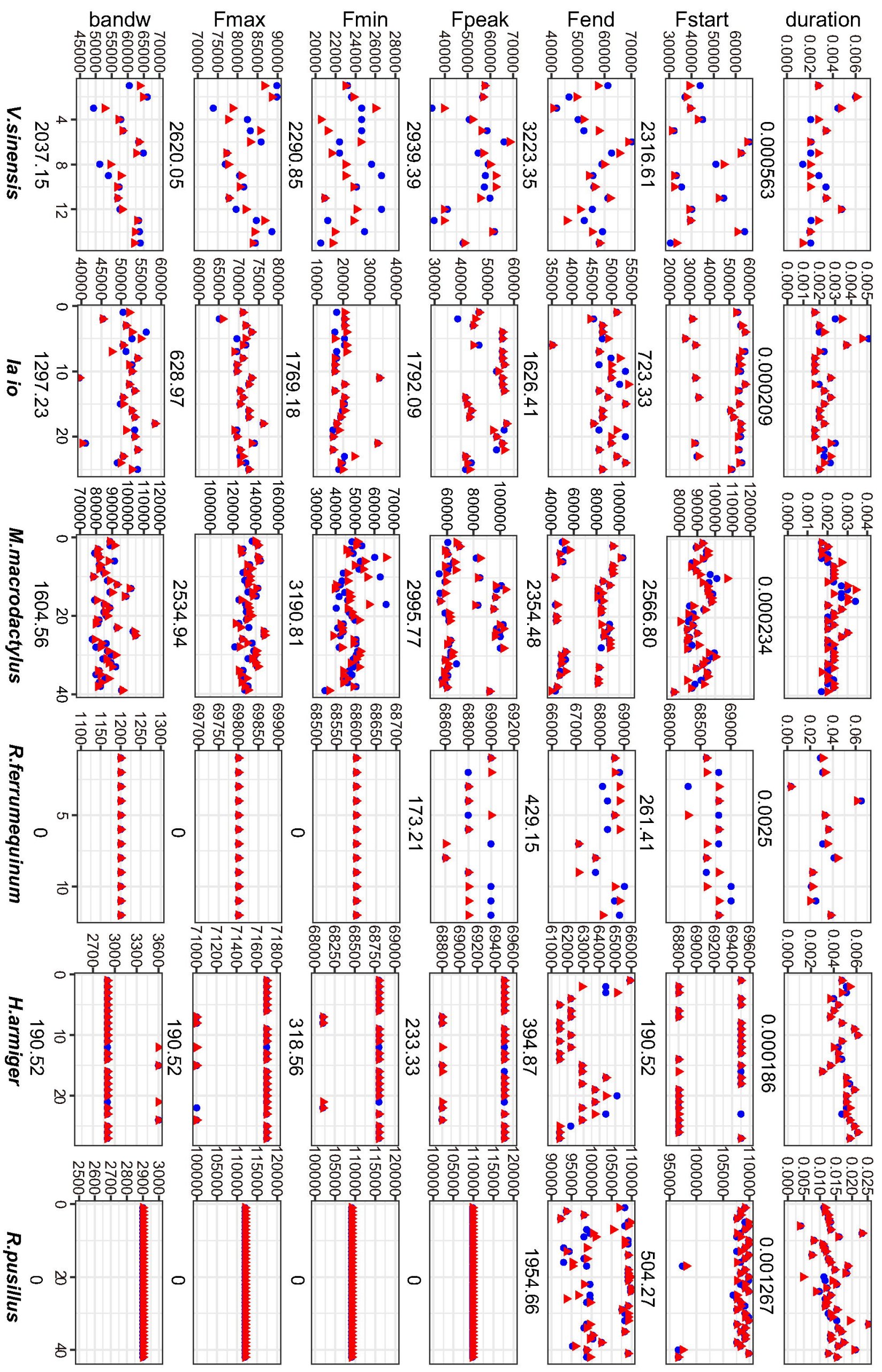


bioRxiv preprint doi: https://doi.org/10.1101/2019.12.15.876656; this version posted December 15, 2019. The copyright holder for this preprint (which was not certified by peer review) is the author/funder, who has granted bioRxiv a license to display the preprint in perpetuity. It is made available under aCC-BY 4.0 International license.

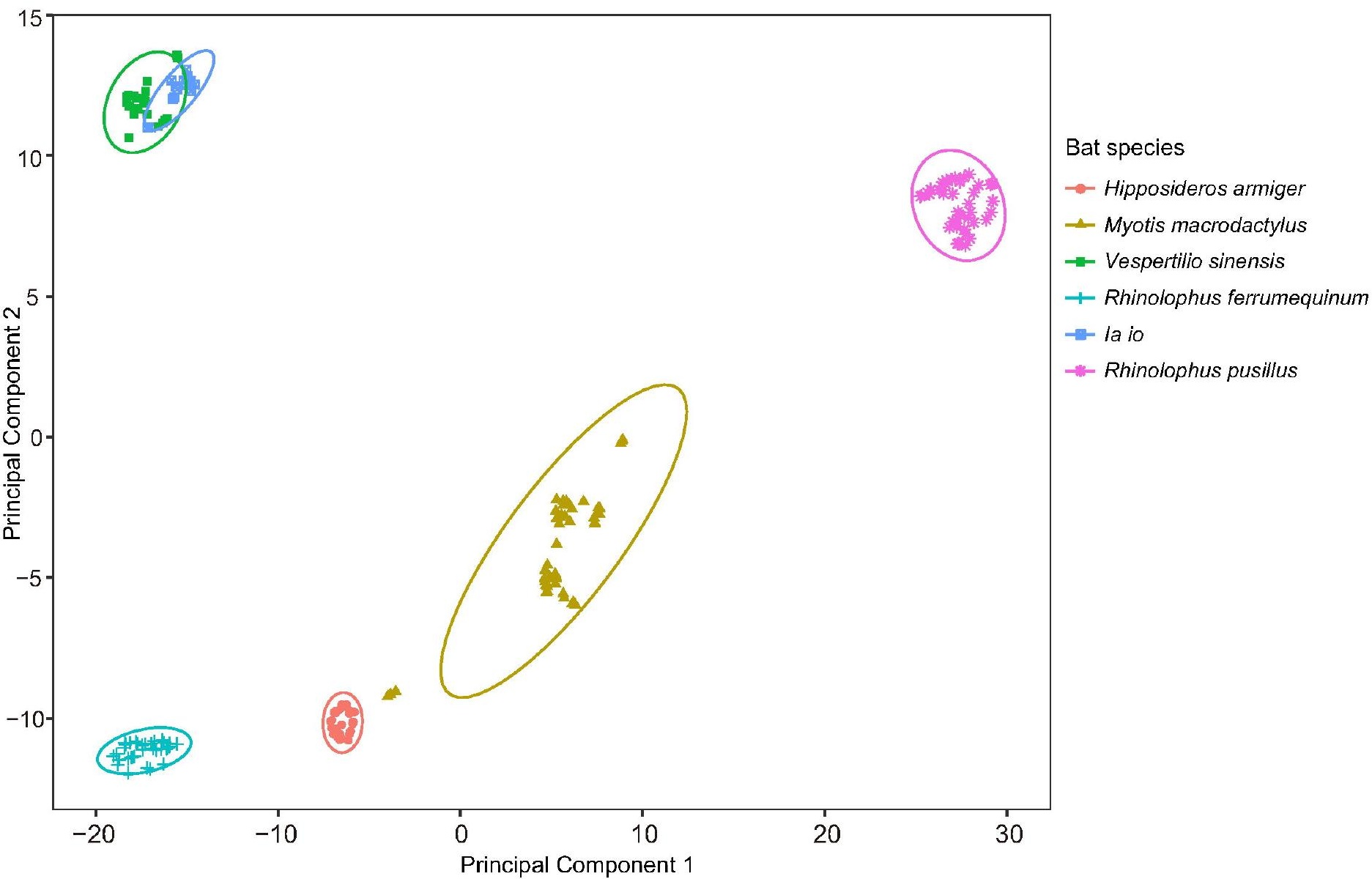


bioRxiv preprint doi: https://doi.org/10.1101/2019.12.15.876656; this version posted December 15, 2019. The copyright holder for this preprint (which was not certified by peer review) is the author/funder, who has granted bioRxiv a license to display the preprint in perpetuity. It is made available under aCC-BY 4.0 International license.

Training

i originally recorded

Overlapping calls

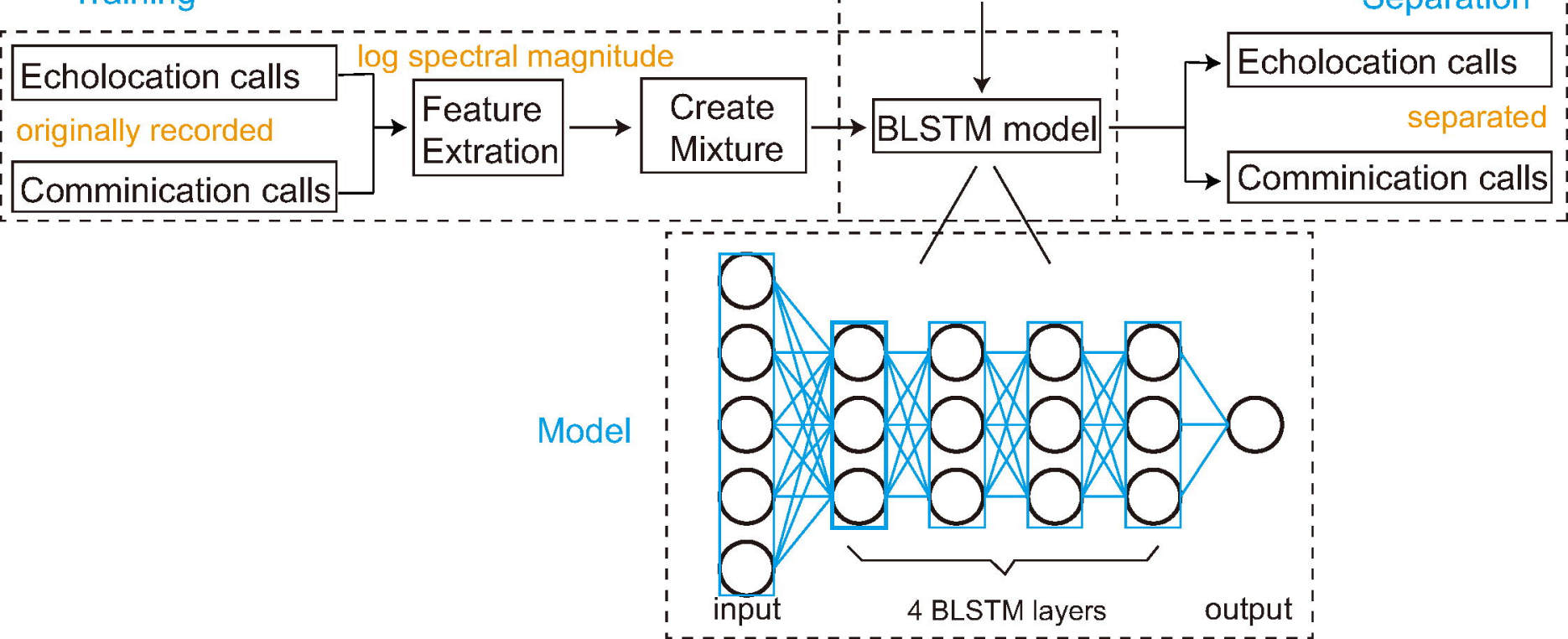


